\title{
Genome wide response to dietary tetradecylthioacetic acid supplementation in the heart of Atlantic Salmon (Salmo salar L)
}

Fabian Grammes ${ }^{1,2}$, Kjell-Arne Rørvik ${ }^{1,2}$, Magny S Thomassen ${ }^{1,2}$, Rolf K Berge ${ }^{3}$ and Harald Takle $2,4^{*}$

\begin{abstract}
Background: Under-dimensioned hearts causing functional problems are associated with higher mortality rates in intensive Atlantic salmon aquaculture. Previous studies have indicated that tetradecylthioacetic acid (TTA) induces cardiac growth and also stimulates transcription of peroxisome proliferator activated receptors (PPAR) $\alpha$ and $\beta$ in the Atlantic salmon heart. Since cardiac and transcriptional responses to feed are of high interest in aquaculture, the objective of this study was to characterize the transcriptional mechanisms induced by $\Pi \mathrm{A}$ in the heart of Atlantic salmon.

Results: Atlantic salmon were kept at sea for 17 weeks. During the first 8 weeks the fish received a TTA supplemented diet. Using microarrays, profound transcriptional effects were observed in the heart at the end of the experiment, 9 weeks after the feeding of TTA stopped. Approximately $90 \%$ of the significant genes were expressed higher in the TTA group. Hypergeometric testing revealed the over-representation of 35 gene ontology terms in the TTA fed group. The GO terms were generally categorized into cardiac performance, lipid catabolism, glycolysis and TCA cycle.

Conclusions: Our results indicate that TTA has profound effects on cardiac performance based on results from microarray and qRT-PCR analysis. The gene expression profile favors a scenario of "physiological"Iright hypertrophy recognized by increased oxidative fatty acid metabolism, glycolysis and TCA cycle activity as well as cardiac growth and contractility in the heart ventricle. Increased cardiac efficiency may offer significant benefits in the demanding Aquaculture situations.
\end{abstract}

\section{Background}

High levels of dietary lipids are used in commercial Atlantic salmon diets to promote rapid growth and as a inexpensive source of energy. These high lipid levels may promote excess lipid deposition in the viscera and the muscle, thereby reducing the market quality of the fish. Thus, tetradecylthioacetic acid (TTA: $\mathrm{CH}_{3}-\left(\mathrm{CH}_{2}\right)_{13}-$ $\left.\mathrm{S}-\mathrm{CH}_{2}-\mathrm{COOH}\right)$ has been tested for aquaculture nutrition, initially to increase lipid catabolism and thereby reducing lipid deposition [1]. However, beneficial effects on cardiac growth and disease resistance have also been addressed.

TTA is a modified fatty acid (FA) that possesses a sulfur atom in the $\beta$ position. Like a normal FA, TTA

\footnotetext{
*Correspondence: harald.takle@nofima.no

2 NOFIMA, P.O. Box 5010, N-1432 Aas, Norway

${ }^{4}$ AVS Chile SA, Casilla 300, Puerto Varas, Chile

Full list of author information is available at the end of the article
}

can be converted to co-enzyme A thioester, but further catabolism by $\beta$-oxidation does not occur. This lack of metabolism is likely to determine the biological effects of TTA. Biological effects of TTA have been the focus of extensive research in rodents and also in humans. The most important findings from these experiments are that TTA increases the mitochondrial and peroxisomal $\beta$-oxidation and possesses hypolipidemic effects. In addition, TTA acts as an antioxidant in vivo and can modulate the inflammatory response (reviewed in [2]). Cell culture experiments demonstrated that TTA can act as a ligand for all Peroxisome proliferator activated receptors (PPARs) [3,4], which are ligand-activated transcription factors. Upon ligand activation PPARs heterodimerizes with retinoic- $\mathrm{X}$-acid receptor (RXR) and have been shown to regulate the expression of genes involved in fatty acid 
metabolism, cell differentiation, development and inflammation (reviewed in [5]). Arguably most of the biological effects of TTA are mediated through activation of PPARs.

Studies addressing the biological effects of TTA in Atlantic salmon have demonstrated that TTA increases $\beta$-oxidation in the liver [1] and white muscle [6]. Furthermore, TTA reduces the secretion of triacylglycerides from Atlantic salmon hepatocytes in vitro [7] and has been shown to increase the expression of genes associated with fat metabolism in the liver and the heart ventricle $[1,8]$. Previous results also suggested that TTA stimulates the transcription of PPAR $\alpha$ and $\beta$ in the heart $[8,9]$, thus indicating that TTA affects the metabolism in Atlantic salmon through activation of PPARs, similar to the mechanism known from rodents. Interestingly, this activation of PPARs may have been related to increased survival after a natural outbreak of a heart related viral disease in Atlantic salmon [8,9]. In mammals, cardiac activation of PPARs has yielded substantial attention due to the fact that PPARs have been proven to be major regulators of cardiac metabolism [10-13]. In addition, PPAR agonists have been reported to exert beneficial effects by attenuating the pathogenesis of heart failure and atherosclerosis $[14,15]$.

Poor development of the outer muscle layer, atherosclerosis and metabolic dysfunction have been related to under-dimensioned hearts and reduced cardiac function in Atlantic salmon aquaculture, consequently resulting in increased mortality [16]. Therefore, methods to improve cardiac metabolism and performance in fish are needed; something which has been sparsely studied. It appears that, similar to the mammalian heart, the oxidative cardiac metabolism in fish depends on the metabolism of fatty acids and glucose [17].

This study aims to characterize the cardiac transcriptional response of Atlantic salmon to a TTA supplemented diet. A feeding trial was conducted in sea, feeding a control and TTA supplemented diet during the first 8 weeks and only control diet for the subsequent 9 weeks of the experiment. Fish were sampled both at the end of the TTA feeding period (8.weeks) and at the end of the experiment (17.weeks). Our results show that administration of TTA to Atlantic salmon resulted in a marked change of cardiac gene expression. The expression profile suggests that TTA induces cardiac fatty acid oxidation, glycolysis, TCA cycle and contractility as well as cardiac growth.

\section{Results}

\section{Production data}

Atlantic salmon that were fed with $0.25 \%$ TTA had significantly lower fat content in the muscle at the 8.weeks sampling point, and showed a tendency for increased mean relative heart weight (Table 1). No significant effect of TTA on fish weight was detected. During the experiment none of the dietary groups showed higher mortality than the control group. In the heart ventricles, 120.2 $\mu g T T A / g$ Tissue was detected at the 8.weeks sampling point in the TTA group, while $1.2 \mu g T T A / g T i s s u e$ was detected in the control group. Based on the TTA measurements of a group fed a higher $(0.5 \% \mathrm{w} / \mathrm{w})$ TTA diet from the same trial, we can assume that the TTA levels in the heart ventricles at the 17.weeks sampling point were no different to the control group (see Additional file 1: Table S1).

\section{Microarray analysis}

RNA cardiac samples from six individual fish from each dietary group and sampling point were used in the microarray analysis, utilizing the Atlantic salmon SIQ2 microarray [18] in a one-color setup, resulting in a total of 24 arrays. After normalization and filtering, 13166 probes $(63 \%)$ were classified as present. To obtain a global overview of the general structure of the dataset we applied correspondence analysis (CA) as an explorative technique

Table 1 Effect of TTA on Atlantic salmon production parameters

\begin{tabular}{|c|c|c|c|c|c|}
\hline & \multirow[t]{2}{*}{ Start sampling } & \multicolumn{2}{|c|}{ 8.weeks } & \multicolumn{2}{|c|}{ 17.weeks } \\
\hline & & Control & $0.25 \%$ TTA & Control & $0.25 \%$ TTA \\
\hline Weight[g] & $102 \pm 5$ & $166 \pm 1$ & $165 \pm 1$ & $438 \pm 4$ & $440 \pm 2$ \\
\hline $\mathrm{CF}^{1}$ & $1.2 \pm 0.1$ & $1.1 \pm 0.1$ & $1.1 \pm 0.1$ & $1.2 \pm 0.1$ & $1.2 \pm 0.1$ \\
\hline$L L^{2}$ & $0.82 \pm 0.05$ & $1.00 \pm 0.02$ & $1.07 \pm 0.07$ & $1.43 \pm 0.04$ & $1.41 \pm 0.04$ \\
\hline $\mathrm{CSI}^{3}$ & $0.074 \pm 0.002$ & $0.092 \pm 0.001$ & $0.094 \pm 0.001$ & $0.101 \pm 0.002$ & $0.103 \pm 0.002$ \\
\hline Mortality[\%] & & $0.74 \pm 0.18$ & $0.41 \pm 0.27$ & $2 \pm 0.23$ & $2 \pm 0.21$ \\
\hline Muscle fat content ${ }^{4}[\%]$ & & $4.2^{a} \pm 0.1$ & $3.9^{b} \pm 0.1$ & $6.5 \pm 0.4$ & $6.8 \pm 0.1$ \\
\hline
\end{tabular}

Values within the same row with different subscripts are significantly different ( $p \leq 0.05, \mathrm{t}$-test). Mean $\pm \mathrm{SEM}$, the statistical unit is the mean of the net pen ( $n=3$ ). 19 fish were used to calculate the net pen mean.

${ }^{1}$ Condition factor $(C F)=\frac{1000 \times \text { weight }[g]}{\text { length }[g]^{3}}$.

${ }^{2}$ Liver index $(\mathrm{LI})=\frac{\text { liver-weight }[g]}{\text { weight }[g]} \times 100$

${ }^{3}$ Cardio somatic index $(\mathrm{CSI})=\frac{\text { heart }- \text { weight }[\text { g }]}{\text { weight }[\mathrm{g}]} \times 100$.

${ }^{4}$ Muscle samples (NQC: Norwegian quality cut) from 10 fish/cage were pooled to analyse the muscle fat content. 
[19]. The first 2 components of the CA are displayed, together explaining $67 \%$ of the total inertia of the different samples (Figure 1). The analysis shows a clear distinction between the two sampling points at 8.weeks and 17.weeks. It further shows a relatively dense cluster at the 8.weeks point with minor separation between samples from the TTA and control group. Even though it was impossible to draw a straight line to separate between the TTA and control samples at the 17.weeks sampling point, we observed a clear tendency of separation.

To identify differentially expressed (DE) probes in the data set we used moderated $t$-statistics [20], comparing samples from TTA to control fed Atlantic salmon for each sampling point.

\section{Sampling point: 8.weeks, end of TTA feeding}

At the 8.weeks sampling point, five genes were found to be DE between the TTA and the control fed group (Figure 2). The genes were: Ephrin-b2, arf gtpase-activating protein (git2), f-box only protein 11 (fbx11), angiopoietinrelated protein 4 (ANGPTL4) and sodium-and chloridedependent creatine transporter 1 (sc6a8). Ephrin-b2 was the only gene found to be down-regulated in the TTA fed group.

Ephrin-b2 (Efnb2) in mammals has been reported to be highly expressed in the heart and serves also as a marker for angiogenesis [21]. The protein GIT-2 participates in pleiotropic cellular processes like cell migration and Tcell activation; however, a function affecting the structure of the cytoskeleton [22] may be relevant in our study. Cardiomyocytes rely solely on the creatine transporter sc6a8 for the uptake of creatine from the plasma. Overexpression of the creatinfabe transporter in mice has been reported to correlate with the myocardial creatine content, but also to be associated with cardiac hypertrophy [23]. Angiopoietin-related protein 4 possesses a role in regulating angiogenesis and is also known as a target gene

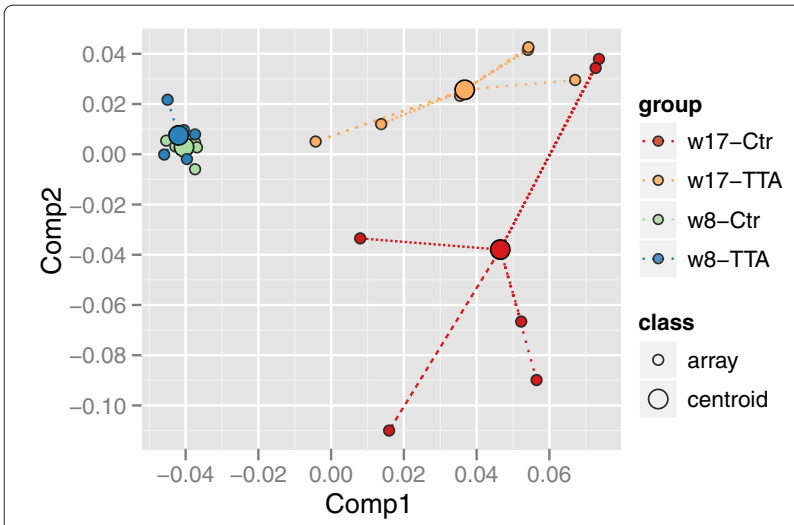

Figure 1 Correspondence Analysis (CA). CA of arrays from the two dietary groups for both sampling points (8.weeks and 17. weeks), $n=6$.

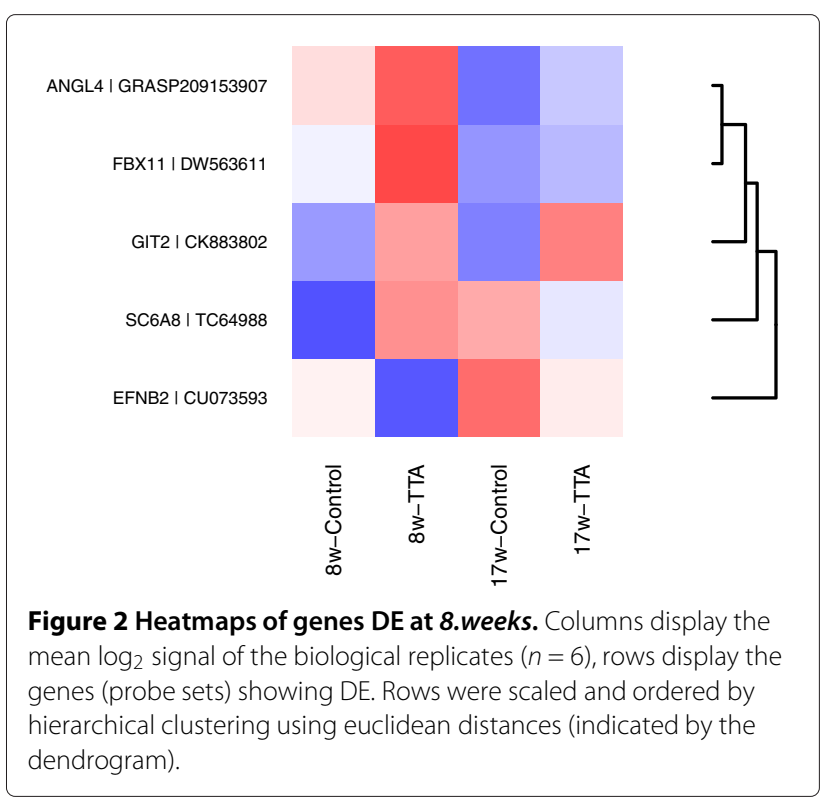

for PPARs and acts as an important stimulator of lipid metabolism [24].

\section{Sampling point: 17 .weeks, 9 weeks post TTA feeding}

1198 probes (930 genes) were found to be DE between the TTA and the control fed group at the 17.weeks sampling point. In order to facilitate a functional interpretation of the vast number of $\mathrm{DE}$ genes, we tested them for enrichment (over-representation) of GO terms from the category "biological process" [25], using conditional hypergeometric testing [26]. To ensure that one gene was represented by a maximum of one probe [26], probes matching the same gene were collapsed prior to hypergeometric testing. This step reduced the total number of probes in the data set from 11143 to 7659 probes and the number of DE probes from 1198 to 930 probes (930 genes). From these 930 genes, $90 \%$ showed higher gene expression in the samples from TTA fed fish.

Conditional hypergeometric testing revealed significant over-representation of 36 GO terms. To simplify interpretation, significant GO terms were grouped into five categories according to their function in the heart (Table2). The grouping was further supported by a strong gene overlap between the different GO terms within the categories (Additional file 2: Figure S1 and Additional file 3: Table S2 ). Overall, the results from the enrichment analysis suggests an increased capacity of heart ventricles from TTA fed Atlantic salmon to catabolize lipids and glycogen. Further, an increased capacity for cardiac contractility and cardiac tissue morphogenesis is indicated.

\section{Heart performance}

The group fed TTA showed an up-regulation in the expression of genes encoding contractile proteins like 
Table 2 Gene Ontology enrichment analysis for sampling point 17.weeks

\begin{tabular}{|c|c|c|c|c|}
\hline GOID & $p$-value & Count $^{1}$ & Size $^{2}$ & Gene ontology term \\
\hline \multicolumn{5}{|c|}{ Fat metabolism } \\
\hline GO:0046395 & $5.33 \mathrm{e}-04$ & 24 & 96 & carboxylic acid catabolic process \\
\hline GO:0034440 & $5.69 e-03$ & 13 & 49 & lipid oxidation \\
\hline GO:0044242 & $8.41 \mathrm{e}-03$ & 19 & 86 & cellular lipid catabolic process \\
\hline \multicolumn{5}{|c|}{ Heart performance } \\
\hline GO:0006936 & $1.56 \mathrm{e}-03$ & 37 & 182 & muscle contraction \\
\hline GO:0008015 & $5.56 \mathrm{e}-03$ & 38 & 202 & blood circulation \\
\hline GO:0003015 & $6.45 e-03$ & 22 & 102 & heart process \\
\hline GO:0008016 & $7.18 \mathrm{e}-03$ & 17 & 73 & regulation of heart contraction \\
\hline GO:0055008 & $8.94 \mathrm{e}-03$ & 12 & 46 & cardiac muscle tissue morphogenesis \\
\hline \multicolumn{5}{|c|}{ Citrate cycle (TCA) } \\
\hline GO:0009109 & $1.87 e-07$ & 15 & 28 & co-enzyme catabolic process \\
\hline GO:0006084 & $2.12 \mathrm{e}-07$ & 18 & 39 & acetyl-CoA metabolic process \\
\hline GO:0006099 & $2.32 \mathrm{e}-07$ & 14 & 25 & tricarboxylic acid cycle \\
\hline GO:0006091 & $9.39 e-05$ & 46 & 214 & generation of precursor metabolites and energy \\
\hline GO:0045333 & $3.49 \mathrm{e}-04$ & 23 & 88 & cellular respiration \\
\hline GO:0044248 & $1.34 \mathrm{e}-03$ & 36 & 180 & cellular catabolic process \\
\hline \multicolumn{5}{|c|}{ carbohydrate metabolism } \\
\hline GO:0006112 & $6.21 e-04$ & 16 & 54 & energy reserve metabolic process \\
\hline GO:0016052 & $1.17 \mathrm{e}-03$ & 19 & 73 & carbohydrate catabolic process \\
\hline GO:0006073 & $1.27 e-03$ & 14 & 47 & cellular glucan metabolic process \\
\hline GO:0046164 & $1.77 \mathrm{e}-03$ & 16 & 59 & alcohol catabolic process \\
\hline GO:0019320 & $2.97 e-03$ & 14 & 51 & hexose catabolic process \\
\hline GO:0006096 & $3.26 \mathrm{e}-03$ & 12 & 41 & glycolysis \\
\hline \multicolumn{5}{|l|}{ Other } \\
\hline GO:0007338 & $1.27 \mathrm{e}-03$ & 14 & 47 & single fertilization \\
\hline GO:0001824 & $1.59 \mathrm{e}-03$ & 12 & 38 & blastocyst development \\
\hline GO:0051246 & $2.00 \mathrm{e}-03$ & 86 & 514 & regulation of protein metabolic process \\
\hline GO:0010171 & $2.83 e-03$ & 20 & 84 & body morphogenesis \\
\hline GO:0055114 & $3.60 \mathrm{e}-03$ & 40 & 210 & oxidation reduction \\
\hline GO:0007050 & $3.67 e-03$ & 19 & 80 & cell cycle arrest \\
\hline GO:0043009 & $5.00 \mathrm{e}-03$ & 48 & 267 & chordate embryonic development \\
\hline GO:0001822 & $5.64 \mathrm{e}-03$ & 19 & 83 & kidney development \\
\hline GO:0040010 & $6.35 e-03$ & 33 & 171 & positive regulation of growth rate \\
\hline GO:0009790 & $7.54 \mathrm{e}-03$ & 124 & 815 & embryo development \\
\hline GO:0001655 & $8.41 \mathrm{e}-03$ & 19 & 86 & urogenital system development \\
\hline GO:0070585 & $8.44 \mathrm{e}-03$ & 10 & 35 & protein localization in mitochondrion \\
\hline GO:0044265 & $8.45 e-03$ & 66 & 399 & cellular macromolecule catabolic process \\
\hline GO:0007018 & $9.35 e-03$ & 16 & 69 & microtubule-based movement \\
\hline GO:0006839 & $9.35 e-03$ & 16 & 69 & mitochondrial transport \\
\hline GO:0006402 & $9.66 \mathrm{e}-03$ & 13 & 52 & mRNA catabolic process \\
\hline
\end{tabular}

${ }^{1}$ Number of times the GO term is represented in the list of DE genes, only GO terms having $\geq 10$ genes were considered.

${ }^{2}$ Number of times the GO term is represented in the filtered list of genes on the array. 
myosin heavy chain 6 (MYH6), myosin light chain (MYL9), cardiac myosin binding protein (MYBPC3), cardiac troponin (TNNT2), myomesin-1 (Myom1) and actin (ACTA1, ACTA2).

Moreover, the same group showed increased expression of the cardiac homeodomain factor $N k x 2.5$ and the iroquois-related homeobox factors 3 and 5 (irx3, irx5). Nkx2.5 and the iroquois transcription factors have been reported to control cardiac morphogenesis and growth $[27,28]$. Furthermore, we observed increased expression of FK506 binding protein $1 A$ and $1 B$ (FKBP1A, FKBP1B) and $\mathrm{Na}^{+} / \mathrm{K}^{+}$-transporting ATPase subunit $\alpha 3$ (ATP2A2), encoding an ion-pump responsible for establishing and maintaining the electrochemical gradients at the plasma membrane of the cardiomyocyte. Decreased amounts of this transporter were found in biopsies from humans suffering heart failure [29]. FKBP1A and B are known to interact with intracellular calcium-release channels. In cardiomyocytes FKBP1B is a binding partner for the major $\mathrm{Ca}^{2+}$ release channel ryanodine receptor 2 (RyR2). RyR2 is required for the $\mathrm{Ca}^{2+}$-induced $\mathrm{Ca}^{2+}$ release from the sacroplasmatic reticulum (SR) causing activation of the contractile proteins. Binding of FKBP1B to RyR2 results in channel closure. Mice deficient for FKBP1B showed no divergence in the normal cardiac phenotype under normal conditions but showed exercise-induced arrhythmias [30]. In relation to $\mathrm{Ca}^{2+}$ signaling, we also found the $\mathrm{Na}^{+} / \mathrm{Ca}^{2+}$ exchanger $S L C 8 A 1$ to be up-regulated. We also found an increased expression of the $\mathrm{SR} \mathrm{Ca}^{2+}$ ATPase 2 (ATP2A2 also known as SERCA2), encoding an SR calcium pump that is a key component of the cardiac excitation-contraction mechanism [31].

The Kv channel interacting protein 1 (Kcnip1) was found to be down-regulated. The protein Kcnip1 is an integral part of the multimeric Kv4 channel complex, and important for modulating the K-flux across this channel by causing a shortening of the cardiac action potential [32]. Prolongation of the cardiac action potential on the other hand, potentially caused by decreased Kcnip1 expression, is associated to cardiac hypertrophy [33].

In summary, the results suggest an increased cardiac hypertrophy together with increased potential for cardiac contractility, as indicated by the higher transcription of the various ion channels/pumps and contractile proteins.

\section{Fat metabolism}

Nearly all of the genes in this group were up-regulated (Figure 3). We found up-regulation of the mitochondrial trifunctional protein $H A D H A$, the mitochondrial fatty acid transporter carnitine palmitoyltransferase (Cpt1a), lipoprotein lipase ( $L p l$ ), the mitochondrial acyl-CoA dehydrogenases ( $A C A D S, A C A D V$ and $A C A D S B$ ) and of peroxisomal multi-functional enzyme type 2 (Hsd17b4). We also observed an up-regulation of malonyl-CoA decarboxylase (MLYCD). Malonyl-CoA is a potent inhibitor of CPT1 and thus crucial in regulating the transport of fatty acids into the mitochondria for catabolism. Malonyl-CoA decarboxylase has been reported to function as a positive regulator of cardiac fatty acid oxidation by decreasing the levels of the CPT1 inhibitor malonyl-CoA [34]. Thus, the results indicate increased fatty acid oxidation capacity in cardiac ventricles from TTA fed Atlantic salmon.

\section{Glycolysis}

The genes of the six GO terms that were grouped together contained almost entirely genes encoding enzymes or subunits participating in glycolysis. We found increased expression of hexokinase 1 (HXK1), the phospho-fructokinases aldolase $A(A L D O A)$ and 6phosphofructokinase type $C$ (K6PP), glycerol-3-phosphate dehydrogenase (GPDA/GAPDH) and the pyruvate dehydrogenases $D L A T$ and $O P D X$. In accordance, we also observed an increased expression of $M L X$-interacting protein $(M L X I P)$, which has been suggested to be an essential regulator of cellular glycolysis [35]. All of the genes showed increased transcription, therefore clearly indicating increased glycolysis in the hearts from TTA fed Atlantic salmon.

\section{Tricarboxylic acid (TCA) cycle}

As in the previously described categories, almost all of the genes in this category showed an increased expression. The proteins encoded by nearly all of the genes in this category are part of the TCA-cycle. For a graphical representation of the genes within the TCA cycle, see Additional file 4: Figure S2.

\section{qRT-PCR}

To validate the microarray results, six genes were analyzed by qRT-PCR between the TTA and control fed group for the 8.weeks and 17.weeks samples, using the same RNA samples that were used in the microarray experiment. The results showed a significant correlation between the logFCs obtained by qRT-PCR and those obtained by microarray (Pearson correlation $r=0.8314 ; p=0.0008$; Figure 4).

In addition, we measured the gene expression of the three PPAR subtypes $\alpha, \beta$ and $\gamma$ in control and TTA fed Atlantic salmon in the four different tissues: Heart, muscle, liver and gut (pyloric caeca) from both sampling points (Figure 5A). Analyzing the expression levels using analysis of variance (ANOVA) showed that only the PPAR $\alpha$ expression in the heart was significantly increased in the TTA group. The gene expression levels of PPAR $\gamma$ in heart and muscle were too low to allow reliable quantification. Since the microarray data revealed quite clearly that TTA affected genes 


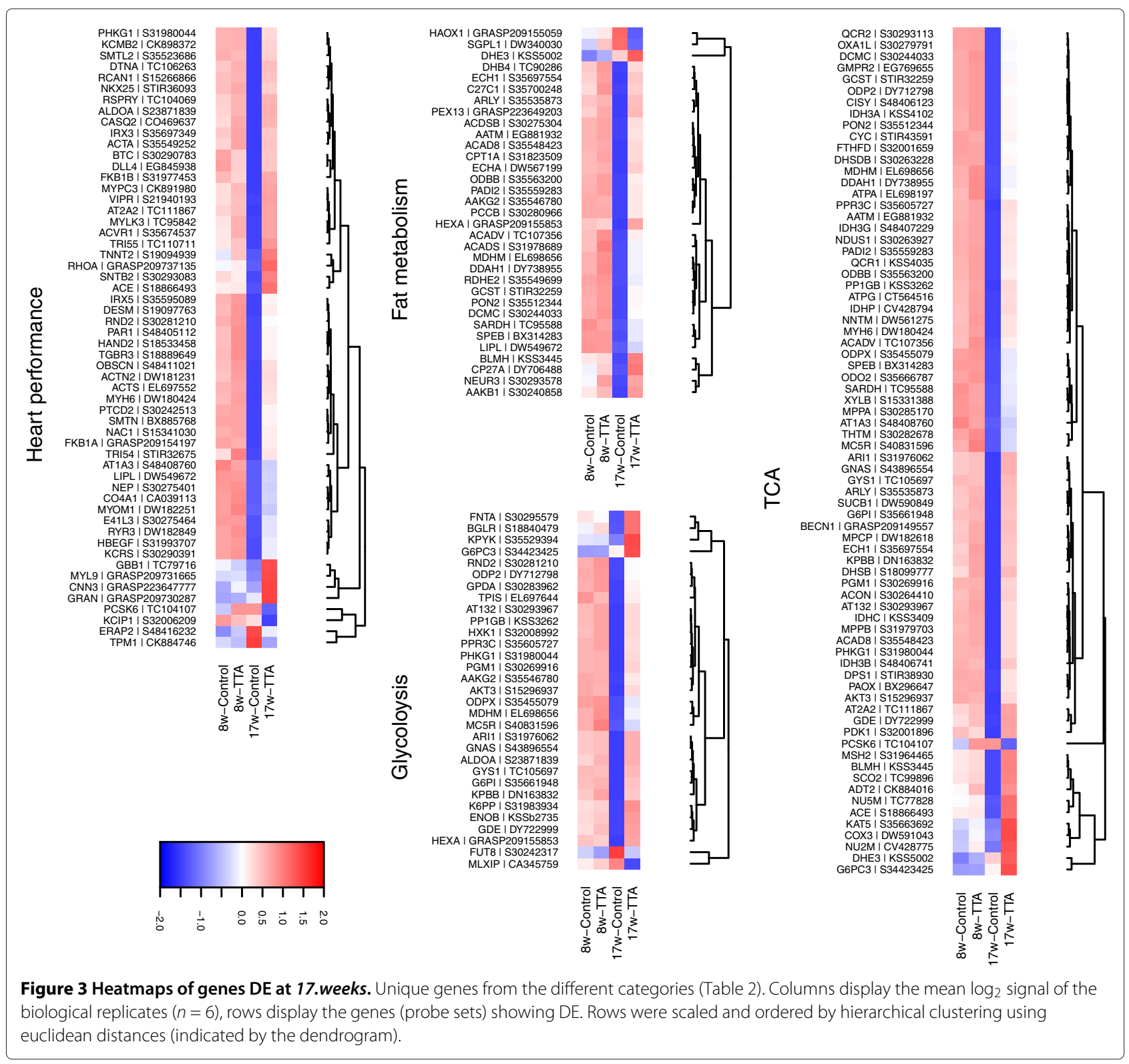

are involved in regulating the heart performance, we used qRT-PCR to measure the expression of the cardiac transcription factors GATA4, Mef2C and osteonectin $(O s x)$. For all three transcription factors we observed a trend of higher mean transcription in the TTA group (Figure 5B), however, only $M e f 2 C$ showed statistical significance.

TTA has previously been reported to stimulate mitochondrial biogenesis in mammals [36]. In this study TTA had no effect on mitochondrial biogenesis as measured by the ratio of $\mathrm{mt} / \mathrm{nDNA}$ (Figure 6). In the liver we found a significant interaction between dietary treatment and time for mitochondrial biogenesis.

\section{Discussion}

In the present study we investigated the response of Atlantic salmon to TTA during the seawater phase. The results from our study show that feeding TTA had profound effects on the cardiac gene expression at sampling point 17.weeks, 9 weeks after TTA feeding ended. The level of TTA applied in the study was, with $0.25 \%$, lower than previous studies that have been conducted in Atlantic salmon (compare [1,6,37]). The mortality rates previously observed in Atlantic salmon in response to high TTA levels was not observed in this study.

Gene set over-representation of the transcription profile at 17.weeks shows an increased capacity of fat catabolism, 


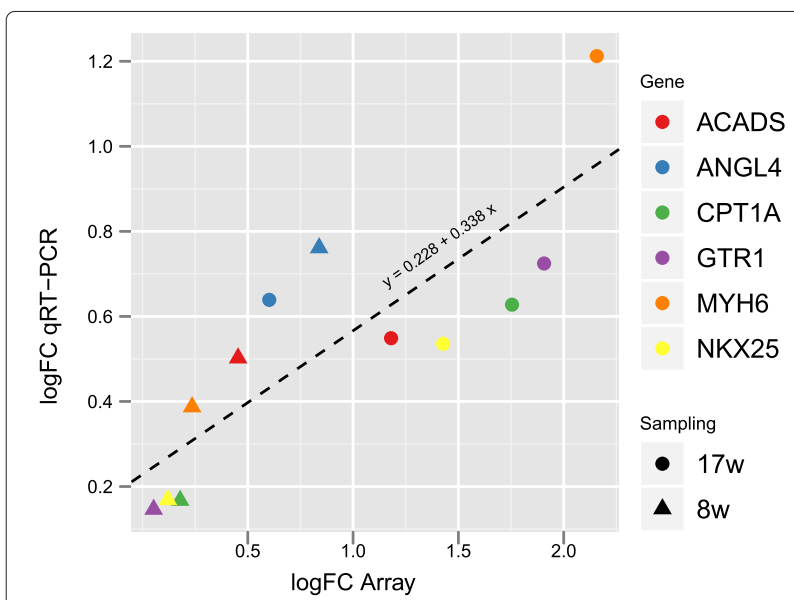

Figure 4 qRT-PCR verification. Comparison of the $\log _{2}$ FC data for the two sampling points between Microarray and qRT-PCR measured samples. qRT-PCR data was normalized to the expression of the housekeeping gene EF1 $\alpha$. Pearson correlation; $r=0.8314, p=0.0008$. The dotted line indicates the linear regression line.

glycolysis and activity of the TCA cycle as well as cardiac contractility and cardiac hypertrophy. Overall, the results suggest a scenario where cardiac ventricles of TTA pre-fed fish are able to generate more energy via a TCA-cycle that is fueled by metabolites from fat catabolism and glycolysis. TTA functions as a ligand for all three PPAR subtypes $[3,4]$, which have crucial functions in the transcriptional regulation of cardiac metabolism. In mice the transcriptional effects of TTA in the heart have been shown to be mediated almost exclusively via PPAR $\alpha$ [38]. Gainof-function and loss-of-function mutations have shown that PPAR $\alpha$ is a crucial transcription factor in the cardiac metabolism, regulating mainly cardiac fatty acid uptake and oxidation $[10,11]$. Furthermore, activation of PPAR $\alpha$ has been demonstrated to shift cardiac energy utilization away from glucose and towards fatty acid oxidation, actually mimicking the cardiac phenotype observed in diabetic hearts [11]. Interestingly the cardiac phenotype of $\operatorname{PPAR} \beta$ differs from that of $\operatorname{PPAR} \alpha$, indicating that both transcription factors regulate, at least partly, different subsets of genes in the heart. PPAR $\beta$ loss-of-function hearts suffer from myocardial lipid accumulation and cardiomyopathy [13]. Gain-of-function mutations on the other hand clearly show that $\operatorname{PPAR} \beta$ positively regulates cardiac glucose utilization [12], and also stimulates cardiac growth [39]. Thus, the significantly higher cardiac transcription of PPAR $\alpha$ and the elevated mean transcription of $\operatorname{PPAR} \beta /$ in concert with the activation of their down-stream pathways, fat catabolism and the glycolysis pathway suggest that cardiac effects of TTA in Atlantic salmon are mediated by both PPAR $\alpha$ and PPAR $\beta$. Intriguingly, over-expression of a constitutively active form of $\operatorname{PPAR} \beta$ in murine skeletal muscle has been reported to mimic training-based muscle adaptation [40]. Hence, it has been speculated, in accordance with the results from $\operatorname{PPAR} \beta$ over-expression in mice [12], that $\operatorname{PPAR} \beta$ causes "physiological" cardiac hypertrophy [39].

Between the 8.weeks and 17.weeks sampling points, the hearts grew by a considerable portion in absolute and relative terms. The gene expression profile in hearts of TTA fed fish at 17.weeks suggests that the cardiac growth of TTA fed fish is shifted towards "physiological" hypertrophy, which may translate to an increased cardiac output. This notion is supported by the expression profile found for the category "cardiac performance" at 17.weeks, unanimously pointing to an increased cardiac contractility and also showing up-regulation of crucial cardiac transcription factors. In particular the higher transcription of the cardiac transcription factors $\mathrm{Nkx} 2.5$ and Mef2C can be regarded as markers for cardiac hypertrophy/growth. It has been demonstrated in mice that over-expression of $\mathrm{Mef} 2 \mathrm{C}$ is sufficient to induce cardiac hypertrophy [41]. Furthermore, both Mef2C and Nkx2.5 have been shown, in vitro, to be regulated by PPAR $\alpha$ in cardiomyocytes [42].

It should also be noted that although we did not find significant differences in relative heart weight in this study, in other studies we found that TTA significantly increases heart size in Atlantic salmon [8,9], and that the effect seems to be correlated to the dose of TTA (Rørvik, unpublished data). Thus, it is tempting to speculate that the increase in relative heart weight may be related to the cardiac transcriptional changes induced by TTA. A "cardiac exercise" stimulating effect is of high relevance for salmonid aquaculture. Atlantic salmon, having a circulatory system that is naturally adapted to long migration routes and high activity, show alteration in cardiac morphology and a reduced relative heart weight in captivity [43]. In addition, circulatory failure has been identified as an important cause of mortality in salmon farming [44]. Thus, using TTA may be one way to support the cardiac performance of fish in captivity.

The highest tissue concentrations of TTA in Atlantic salmon, as well as in mice, can be found in the heart $[1,45]$. In accordance, the heart was also the tissue where the strongest transcriptional response of $\operatorname{PPAR} \alpha$ was detected. The main transcriptional effects were found nine weeks after the TTA feeding stopped and where our data suggested that the cardiac tissue levels of TTA were neglectable. However, we have no information about the course of gene expression between both sampling points, thus it might very well be that the effects of sampling at 17.weeks are the remains of earlier, stronger transcriptional effects. It is remarkable that a similar, delayed response in expression of lipid metabolism related genes to TTA has been observed in our previous Atlantic 


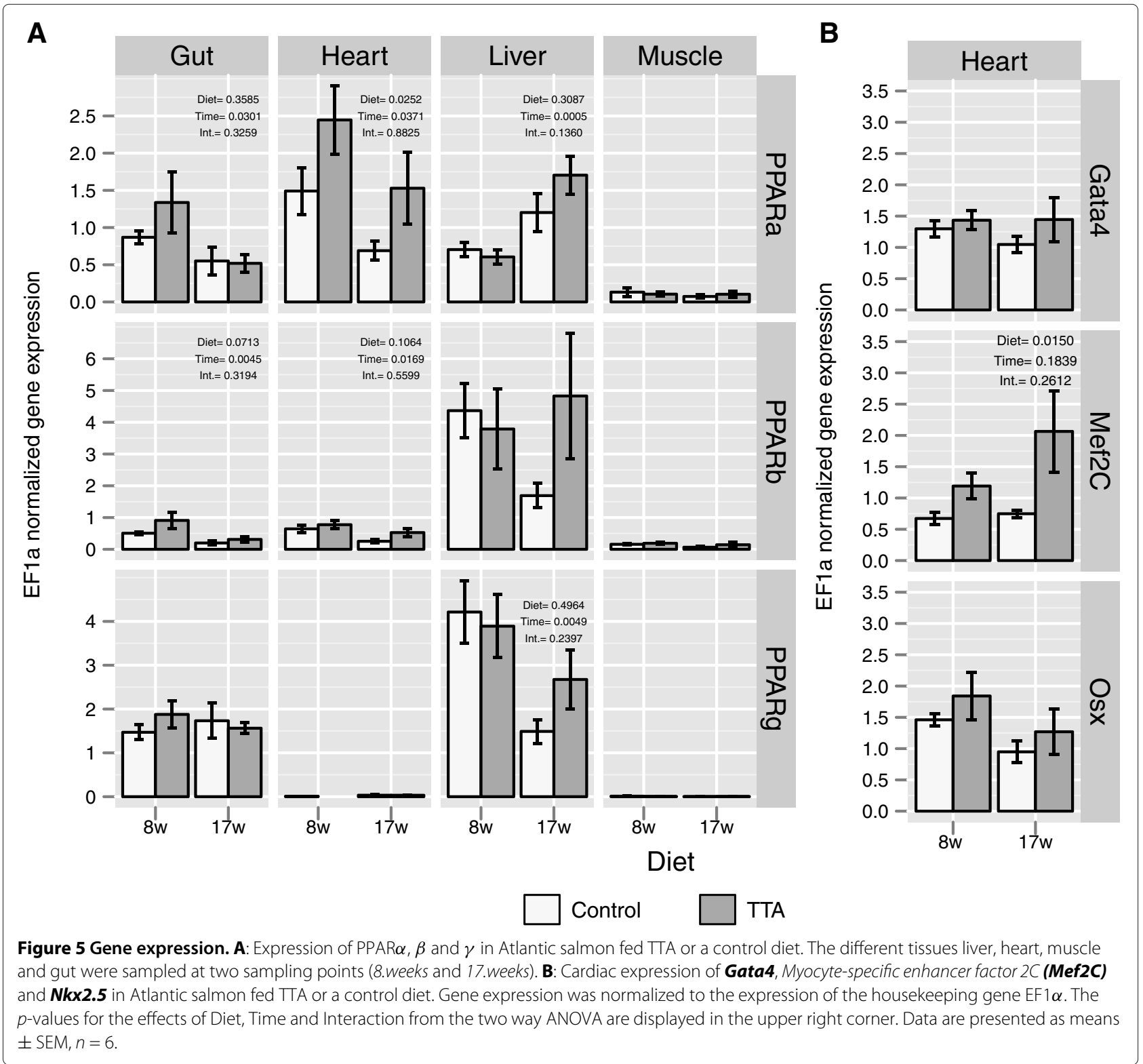

salmon studies [8,9], indicating a common underlying mechanism. It is possible that the delay in transcriptional response is caused by a common, yet unknown, mechanism.

\section{Conclusions}

In conclusion, based on results from microarray analysis, this study demonstrates that TTA increases cardiac fatty acid oxidation and glycolysis as well as contractility and cardiac hypertrophy in Atlantic salmon. The gene expression profiles further favor a scenario of "physiological" hypertrophy in response to TTA. This increased cardiac efficiency may offer significant benefits in situations with increased oxygen demand.

\section{Methods}

Feeding trial

The experiment was conducted at Nofima Marin seawater research station, Averøy, western Norway. Atlantic salmon used in this experiment were hatched at Nofima Marin research station (Sunndaløra, Norway) one year earlier (S1/1+ Salmon). The experiment started with the seawater transfer of the fish on the 15th of May 2007 and lasted until the 27th of September 2007. A randomized block design with triplicate seawater net-pens and 400 fish per pen $\left(\right.$ pen $\left.=125 \mathrm{~m}^{3}\right)$ was used for the experiment. Control and TTA diets $(0.25 \%$ (w/w) TTA (Thia Medica, Norway)) were produced by Biomar (Biomar AS, Myre, Norway). Both TTA and control diets were fed to the fish 


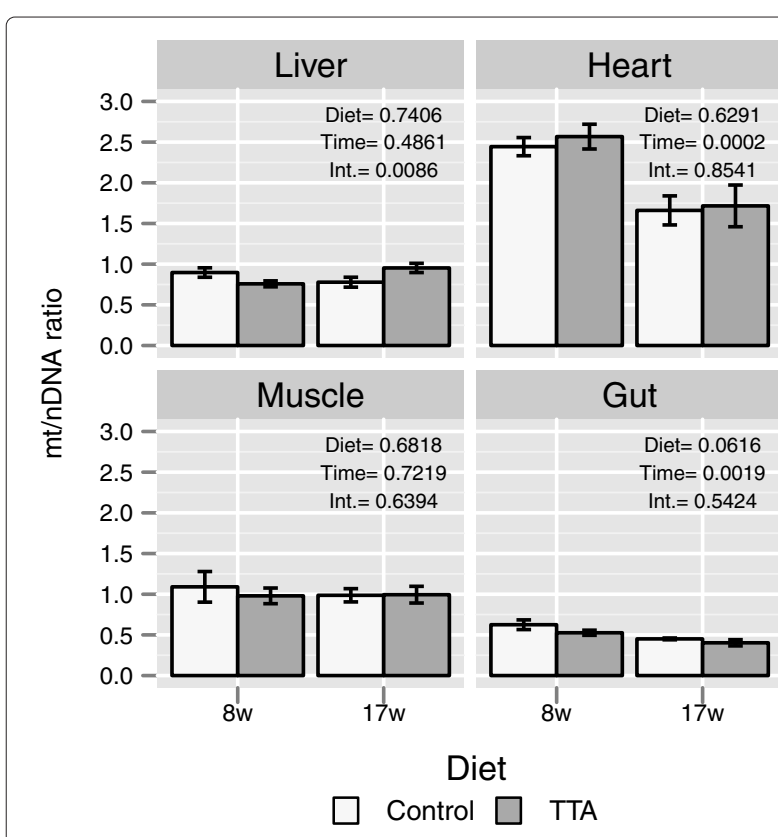

Figure 6 Mitochondrial biogenesis. Effect of feeding TTA on the ratio between mitochondrial and nuclear DNA in the different tissues. The p-values for the effects of Diet, Time and Interaction from the two way anova are displayed in the upper right corner. Data are presented as means $\pm \operatorname{SEM}, n=6$.

until the 16th of July 2007 , from this point until the end of the experiment only the control diet was fed to the fish. Low levels of TTA $(0.25 \%)$ and a short feeding period were chosen in order to avoid negative TTA effects (mortality, altered kidney morphology $[1,46])$. TTA was fed for the first eight weeks after sea transfer, a period where the physiology of the salmon alters due to changing from a fresh to a saltwater environment; and we speculated that an increased capacity for energy utilization may be beneficial. Fish were sampled from the cages to represent the average fish weight for the cage. Sampling was done on the following dates: 16-18th of July 2007 (sampling point: 8.weeks, end of the TTA feeding period) and 25-27th of September 2007 (sampling point: 17.weeks). For each sampling point, fish were sampled for: heart ventricle, liver, muscle and gut (pyloric caeca). The tissue samples were snap frozen in liquid nitrogen and stored at $-80^{\circ} \mathrm{C}$.

\section{Fat analysis}

Fat content in the muscle (Norwegian quality cut-NQC, Norwegian standard procedure - NS 9401 1994) was measured in pooled samples (10 fish) from each net pen as described in [47]. TTA was measured within the total cardiac lipids. For the analysis 10 ventricles from Atlantic salmon out of the same net pen were pooled. Total heart lipids were extracted with chloroform-methanol [48] and fatty acid methyl esters (FAME) were obtained by heating of lipids with methanol at $90^{\circ} \mathrm{C} / 1$ hour, where $\mathrm{H}_{2} \mathrm{SO}_{4}$ was used as a catalyst [49]. After extraction into an organic solvent, the FAME were analyzed by gas-liquid chromatography. A gas chromatograph GC 8000 TOP (Finnigan, USA) was equipped with a programmed temperature vaporization (PTV) injector, flame-ionization detector (FID), AS 800 autosampler and a fused silica capillary column coated with dimethylpolysiloxane stationary phase, DB1-ms (J \& W Scientific, USA). Hydrogen was used as a carrier gas. Column temperature was programmed from 110 to $310^{\circ} \mathrm{C}$ with a gradient $2.5^{\circ} \mathrm{C} / \mathrm{min}$. The GC signal was acquired using Chromeleon software (Dionex, USA). Peaks were identified by means of known FA standards (Larodan Fine Chemicals, Sweden and Sigma-Aldrich, USA) and by means of mass spectra, obtained by GC/MS analysis (GCQ, Finnigan, USA) on the same column. An internal standard (C21:0) was used for quantitation after calibration with known mixtures of FA standards.

\section{RNA extraction}

Two individual samples from each one of the 3 net-pens were samples for heart ventricle, liver, muscle and gut. The samples were randomly chosen $(n=6$ per dietary group and sampling point) and homogenized using a rotor tissue lyser (Precellys 24, Bertin technologies, France). Total RNA was extracted and purified using column purification (96 universal Tissue Kit, Qiagen, Hilden, Germany) according to the manufacturer's instructions. Traces of genomic DNA in the samples were eliminated by oncolumn-DNase digestion (Qiagen). RNA concentrations were measured for all samples using a NanoDrop 1000 Spectrophotometer (Thermo Fisher Scientific, Wilmington, USA). RNA quality for samples later used in the microarray was determined using a Agilent 2100 Bioanalyzer (RNA 6000 NanoLabChip, Agilent, Waldborn, Germany).

\section{Microarray hybridization}

A customized oligo (60-mer) Atlantic salmon microarray in the $4 \times 44 \mathrm{~K}$ format (Agilent, [18]) was used to detect differential gene expression between samples from the heart ventricles of control and $0.25 \%$ TTA fed fish for the 8.weeks and 17.weeks sampling points. The array contained 21012 different probes spotted in duplicates. RNA samples from individual fish were hybridized to the microarray. 24 individual microarrays were performed using 12 fish (6 control and 6 TTA fish) at the 8 week sampling point, and similarly at the 17 week sampling point. All RNA samples used in the hybridization had RIN values ranging from 9.5 to 10. 500ng RNA were amplified and labeled with Cy3 using the Quick Amp Labeling Kit (One Color-Agilent). After purification the cRNA was 
quantified using NanoDrop. Subsequently $1.65 \mu \mathrm{g}$ Cy-dyelabeled cRNA was fragmented (mean size, approximately 50-100 nucleotides) with fragmentation buffer (Agilent Technologies) at $60^{\circ} \mathrm{C}$ for $30 \mathrm{~min}$; cRNA was subsequently hybridized to the microarray at $65^{\circ} \mathrm{C}$ for $17 \mathrm{~h}$. All steps were conducted according to the Agilent protocol (OneColor Quick Amp Labeling, Version 5.7). The microarray chips were scanned using a Agilent Microarray Scanner (G2565CA) and analysis of the microarray images was done in Agilent's Feature Extraction Software (Version 10.5.1.1) using the one-color (GE1_105_Dec08) protocol.

\section{Microarray analysis}

Normalization and analysis of the data was performed in R/Bioconductor [50,51] using the "limma" package [20]. The background corrected fluorescence signals (gProcessedSignal) were obtained from Feature Extraction (Agilent). Spots were filtered according to the following criteria provided by Feature Extraction: gIsFound, gIsPosAndSignif and gIsWellAboveBG (a description of the parameters can be found in the Feature Extraction Software Reference Guide). The mean signal of the duplicated probes was calculated and all control spots together with probe sets showing more than three missing values were removed from the dataset. The data was subsequently normalized using quantile normalization in order to adjust the scale of intensities across arrays [52]. After normalization the signals were $\log _{2}$ transformed. The normalized/filtered dataset then contained 13166 probe sets (63\% of the total). The raw and normalized data is publicly available at NCBI's GEO repository (http://www.ncbi.nlm.nih.gov/geo/, AccNr: GSE25305).
Differential expression of probe sets was assessed by fitting a linear model, including the effects of feeding ( 2 levels: Control and TTA) and the effects of sampling point (2 levels: 8.weeks and 17.weeks) and their interaction. The specific comparisons: TTA vs. Control at sampling point 8.weeks and TTA vs. Control at sampling point 17.weeks were made by extracting the appropriate contrasts from the linear model. For each contrast moderated $t$-statistics were calculated using an empirical Bayes method [53]. Probes without annotation were removed from the dataset before controlling the false discovery rate [54] simultaneously across probe sets and contrasts (method: "global" in the limma function "decideTests"). Probe sets with a $q$-value $\leq 0.05$ and a $\log _{2} \mathrm{FC} \geq 0.5$ were declared $\mathrm{DE}$ for the corresponding contrast. A comprehensive list of all DE probes for each contrast can be found in Additional file 5: Table S3.

Probe annotation and GOs were retrieved using the top Blast function implemented in Blast2GO [55]. Full length probe sequences were blasted against protein sequences from the Swissprot database in a BlastX search. The E-value cut off was set to $10^{-6}$. Hypergeometric testing for over-representation of GO terms from the category biological process [25] among the genes DE for the contrast TTA vs. Control at the 17.weeks sampling point was conducted using the GOstats package [26]. Before testing, probes matching to the same gene were collapsed to the probe showing the largest variance.

Correspondence analysis was conducted using the $\mathrm{R}$ package "made4" [56]. Probe sets with missing values

Table 3 qRT-PCR primer sequences

\begin{tabular}{|c|c|c|c|}
\hline Gene & Accession no. & Forward primer $\left(3^{\prime}-5^{\prime}\right)$ & Reverse primer $\left(5^{\prime}-3^{\prime}\right)$ \\
\hline mt D Loop B (gDNA) & NC001960 & CCCCTGAAAGCCGAATGTAA & CGACCTTGTTAGACTTCTITGCTTG \\
\hline MyoD2 (gDNA) & AJ557150 & CAGAGCCAGGATTACACTCGTTACA & GCATGTCGCTGGTGTTGAAG \\
\hline $\operatorname{PPAR} \alpha$ & DQ294237 & TCCTGGTGGCCTACGGATC & CGTTGAATTCATGGCGAACT \\
\hline $\operatorname{PPAR} \beta$ & AJ416953 & GAGACGGTCAGGGAGCTCAC & CCAGCAACCCGTCCTTGTT \\
\hline PPAR $\gamma$ & AJ416951 & CATTGTCAGCCTGTCCAGAC & TTGCAGCCCTCACAGACATG \\
\hline $\mathrm{EF} 1 \alpha$ & AF321836 & CACCACCGGCCATCTGATCTACAA & TCAGCAGCCTCCTTCTCGAACTTC \\
\hline MYH6 & DW559270 & CAGGTCCTCTATGTGCTGGTGTG & TCCTCATTGTAGTTGCTGTCCTCAC \\
\hline ANGL4 & GRASP209147493 & CCGTATGGGGGATGATGCTAA & GGTAGTATGCTGACGACTGACACCT \\
\hline GTR1 & S30269700 & GCCATGGATGTCCTACGTGA & CTCCGCTACATACGGGAAGG \\
\hline CPT1A & S31823509 & TCCCACATCATCCCCTTCAACT & TGTCCCTGAAGTGAGCCAGCT \\
\hline ACADS & S31978689 & CTGGGGAAGAAGGAGGACAAG & TCTAGAGCAGCCTGAGCAATACC \\
\hline NKX2.5 & DW550500 & CCCAGTACGTCCACACCCTT & GGAGGTCGGTAAGGCACAGT \\
\hline Mef2C & GU252207 & CACCGTAACTCGCCTGGTCT & GCTTGCGGTTGCTGTTCATA \\
\hline GATA4 & HM475152 & TCTCCATTCGACAGCTCCGT & CATCGCTCCACAGTTCACACA \\
\hline Osx & FJ195614 & ATTACTGAGGAGGAGCCCATCATT & CCTCATCCACCTCACACACCTT \\
\hline
\end{tabular}


were removed from the dataset prior to correspondence analysis.

\section{Quantitative RT-PCR}

Single strand cDNA was synthesized from 500ng of total RNA using oligo dT primers and the Taq Man reverse transcription Kit (Applied Biosystems, CA, USA). qRT-PCR was performed on a Light-Cycler 480 (Roche, Switzerland). For the PCR reaction, $2 x$ SYBR green I master Mix (Roche), $0.41 \mathrm{nM}$ of each primer and the cDNA template were mixed in a total reaction volume of $10 \mu \mathrm{l}$. Primer sequences are listed in Table 3. A three step PCR protocol with 45 cycles $\left(15 \mathrm{~s} 95^{\circ} \mathrm{C}, 15 \mathrm{~s} 60^{\circ} \mathrm{C}, 15 \mathrm{~s} 72^{\circ} \mathrm{C}\right)$ was used. To verify specific amplification, a melting curve analysis step was done at the end of the program. In order to verify the results obtained through the microarray experiment, the same 24 samples used in the array were used in a qRT-PCR approach. Six genes were then randomly picked and samples were analyzed in duplicates. The expression level was calculated using the standard curve method (Applied Biosystems User Bulletin 2). The standard curve was produced from a serial dilution of a pool consisting of all cDNA samples. The expression levels were standardized to the expression of the housekeeping gene elongation factor $1 \alpha(E F 1 \alpha,[57])$.

\section{$\mathrm{mt} / \mathrm{nDNA}$ ratio}

Genomic DNA was isolated from tissue samples from the same individuals as the ones used for the total RNA extraction (totally 96 samples, $n=6$ ). DNA was isolated using DNAeasy kit (Qiagen) according to the manual. The DNA quality for all samples was checked on a $1 \%$ agarose gel and concentration was measured using a NanoDrop Spectrophotometer. The MyoD gene (intron-exon spanning primers) and the mitochondrial D-loop were amplified by qRT-PCR. For the PCR reaction $1 x$ SYBR green I master Mix (Roche), $0.41 \mathrm{nM}$ of each primer and the 6.4ng DNA template were mixed in a reaction volume of $10 \mu$ l. PCR amplification was conducted as described above. All reactions were run in duplicates. Absolute concentrations for mt- and nDNA samples were obtained using the standard curve method. The ratio was calculated by dividing the absolute mtDNA by the absolute nDNA concentration.

\section{Statistical analysis}

All data are presented as means \pm SEM with an $n$ value as stated. The effect of dietary treatment on the production parameters and qRT-PCR were analyzed by 2-way analysis of variance (ANOVA), using dietary treatment and sampling point as fixed factors and block as a random factor. TTA effects on gene expression and $\mathrm{mt} / \mathrm{nDNA}$ ratio were calculated using $E F 1 \alpha$ standardized expression values in a 2-way ANOVA with dietary treatment and time as fixed factors. Unless otherwise stated the statistical unit is the individual fish. All analyses were conducted using $\mathrm{R}$ [50], plots were produced using the $\mathrm{R}$ package ggplot [58] and the heatmaps were produced with the R package lattice/latticeExtra [59].

\section{Additional files}

Additional file 1: Table S1. TTA measurements in the cardiac ventricles. Additional file 2: Figure S1. Overlap matrix of the genes from the $36 \mathrm{GO}$ terms that were significantly over represented in TTA fed Atlantic salmon at sampling point 17.weeks. Rows and columns are hierarchical clustered (indicated by the dendrogram) based on euclidean distance. Overlap is indicated by red color.

Additional file 3: Table S2. Full list of DE genes associated to over-represented GO Biological processes terms at sampling point 17.weeks.

Additional file 4: Figure S2. KEGG Pathway diagram. Seven genes from the category TCA (Table 2) could be annotated to a KEGG Ontology (KO) using the program KAAS [60]. These 7 genes were highlighted (yellow/red) in the KEGG reference pathway: TCA-cycle (ko:00020). The genes were annotated to the following enzymes: Citrate synthase [EC:2.3.3.1] - 1 gene; Isocitrate dehydrogenase [EC:1.1.1.42] - 1 gene; Isocitrate dehydrogenase (NAD+) [EC:1.1.1.41] - 3 genes; Succinate dehydrogenase (ubiquinone) flavoprotein subunit [EC:1.3.5.1] - 1gene; and membrane anchor unit [EC:1.3.5.1] -1 gene

Additional file 5: Table S3. Full list of the DE genes after collapsing. Column 1: Probe ID; Column 2: $\log _{2} F C$ s of the contrast TTA vs. Control for sampling point 8 . weeks; Column 3: $\log _{2} \mathrm{FCs}$ of the contrast TTA vs. Control for sampling point 17.weeks; Column 4-6: Gene annotation: Gene name, gene symbol and e-value. Column 7-8: Significance of the corresponding gene for the corresponding contrast. Column 9: Joint between GenelD and Symbol, as used in the heatmaps.

Competing interests

The authors declare that they have no competing interests.

\section{Authors contributions}

$H T, K R$ and MT conceived the study and designed the experiment. RB provided the TTA measurements. FG conducted the lab experiment, data analysis and drafted the manuscript. All authors read and approved the final manuscript.

\section{Acknowledgements}

We would like to thank Dr. Aleksei Krasnov for designing the Atlantic salmon microarray. We would also like to thank Thia Medica for supplying the TTA used in this experiment.

\section{Author details}

${ }^{1}$ Institute of Animal and Aquaculture Sciences, Norwegian University of Life Sciences, P.O. Box 5003, N-1432 Ås-UMB, ,Norway. ${ }^{2}$ NOFIMA, P.O. Box 5010, N-1432 Aas, Norway. ${ }^{3}$ Institute of Medicine, Haukeland University Hospital, University of Bergen, N-5021 Bergen, Norway. ${ }^{4}$ AVS Chile SA, Casilla 300, Puerto Varas, Chile.

Received: 30 June 2011 Accepted: 13 April 2012 Published: 11 May 2012

\section{References}

1. Moya-Falcón C, Hvattum E, Dyrøy E, Skorve J, Stefansson SO, Thomassen MS, Jakobsen JV, Berge RK, Ruyter B: Effects of 3-thia fatty acids on feed intake, growth, tissue fatty acid composition, beta-oxidation and $\mathrm{Na}+, \mathrm{K}+-\mathrm{ATPase}$ activity in Atlantic salmon. Comp Biochem Physiol B, Biochem Mol Biol 2004, 139(4):657-68.

2. Berge RK, Skorve J, Tronstad KJ, Berge K, Gudbrandsen OA, Grav H: Metabolic effects of thia fatty acids. Curr Opin Lipido/ 2002, 13(3):295-304. 
3. Westergaard M, Henningsen J, Svendsen ML, Johansen $C_{\text {, Jensen UB, }}$ Schrøder HD, Kratchmarova I, Berge RK, Iversen L, Bolund L, Kragballe K, Kristiansen K: Modulation of keratinocyte gene expression and differentiation by PPAR-selective ligands and tetradecylthioacetic acid. J Invest Dermatol 2001, 116(5):702-12.

4. Forman BM, Chen J, Evans RM: Hypolipidemic drugs, polyunsaturated fatty acids, and eicosanoids are ligands for peroxisome proliferator-activated receptors alpha and delta. Proc Natl Acad SC USA 1997, 94(9):4312-7.

5. Berger JP, Akiyama TE, Meinke PT: PPARs: therapeutic targets for metabolic disease. Trends Pharmaco/ Sci 2005, 26(5):244-51.

6. Rørvik KA, Alne H, Gaarder M, Ruyter B, Måseide NP, Jakobsen JV, Berge RK, Sigholt T, Thomassen MS: Does the capacity for energy utilization affect the survival of post-smolt Atlantic salmon, Salmo salar L., during natural outbreaks of infectious pancreatic necrosis? J Fish Dis 2007, 30(7):399-409.

7. Vegusdal A, Gjøen T, Berge RK, Thomassen MS, Ruyter B: Effect of 18:1n-9, 20:5n-3, and 22:6n-3 on lipid accumulation and secretion by Atlantic salmon hepatocytes. Lipids 2005, 40(5):477-86.

8. Alne H, Thomassen MS, Takle H, Terjesen BF, Grammes F, Oehme M, Refstie S, Sigholt T, Berge RK, Rørvik KA: Increased survival by feeding tetradecylthioacetic acid during a natural outbreak of heart and skeletal muscle inflammation in So Atlantic salmon, Salmo salar L. J Fish Dis 2009, 32(11):953-61.

9. Grammes F, Rørvik KA, Takle H: Tetradecylthioacetic acid modulates cardiac transcription in Atlantic salmon, Salmo salar L., suffering heart and skeletal muscle inflammation. J Fish Dis 2012, 35(2):109-17.

10. Sambandam N, Morabito D, Wagg C, Finck BN, Kelly DP, Lopaschuk GD: Chronic activation of PPARalpha is detrimental to cardiac recovery after ischemia. Am J Physiol Heart Circ Physiol 2006, 290:H87-95.

11. Finck BN, Lehman JJ, Leone TC, Welch MJ, Bennett MJ, Kovacs A, Han X, Gross RW, Kozak R, Lopaschuk GD, Kelly DP: The cardiac phenotype induced by PPARalpha overexpression mimics that caused by diabetes mellitus. J Clin Invest 2002, 109:121-30.

12. Burkart EM, Sambandam N, Han X, Gross RW, Courtois M, Gierasch CM, Shoghi K, Welch MJ, Kelly DP: Nuclear receptors PPARbeta/delta and PPARalpha direct distinct metabolic regulatory programs in the mouse heart. J Clin Invest 2007, 117(12):3930-9.

13. Cheng L, Ding G, Qin Q, Huang Y, He N, Evans RM, Schneider MD, Brako FA, Xiao Y, Chen YE, Yang Q: Cardiomyocyte-restricted peroxisome proliferator-activated receptor-[delta] deletion perturbs myocardial fatty acid oxidation and leads to cardiomyopathy. Nat Med 2004 10(11):1245-50.

14. Chinetti G, Fruchart JC, Staels B: Peroxisome proliferator-activated receptors (PPARs): nuclear receptors at the crossroads between lipid metabolism and inflammation. Inflamm Res 2000, 49(10):497-505.

15. Finck BN: The PPAR regulatory system in cardiac physiology and disease. Cardiovasc Res 2007, 73(2):269-77.

16. The health situation in Norwegian aquaculture 2009. Tech. rep. Norwegian Veterinary Institute 2009. Http://www.vetinst.no/eng/ Research/Publications/Fish-Health-Report.

17. Driedzic WR: Cardiac energy metabolism. In Fish Physiology, Volume 7. Edited by Hoar WS, Randall DJ, Farrell AP. New York: Academic Press; 1992:219-266.

18. Krasnov A, Timmerhaus G, Afanasyev S, Jørgensen SM: Development and assessment of oligonucleotide microarrays for Atlantic salmon (Salmo salar L.). Comp Biochem Physiol Part D Genomics Proteomics 2010.

19. Fellenberg K, Hauser NC, Brors B, Neutzner A, Hoheisel JD, Vingron M: Correspondence analysis applied to microarray data. Proc Natl Acad Sci USA 2001, 98(19):10781-6.

20. Smyth GK: Limma: linear models for microarray data. In Bioinformatics and Computational Biology Solutions using R and Bioconductor. Edited by Gentleman R, Carey V, Dudoit S, R Irizarry WH. New York: Springer; 2005:397-420

21. Gerety SS, Anderson DJ: Cardiovascular ephrinB2 function is essential for embryonic angiogenesis. Development 2002, 129(6):1397-410.

22. Hoefen RJ, Berk BC: The multifunctional GIT family of proteins. $J$ Cell Sci 2006, 119(Pt 8):1469-75.

23. Wallis J, Lygate CA, Fischer A, ten Hove, M, Schneider JE, Sebag-Montefiore L, Dawson D, Hulbert K, Zhang W, Zhang MH, Watkins $\mathrm{H}$, Clarke K, Neubauer S: Supranormal myocardial creatine and phosphocreatine concentrations lead to cardiac hypertrophy and heart failure: insights from creatine transporter-overexpressing transgenic mice. Circulation 2005, 112(20):3131-9.

24. Mandard S, Zandbergen F, van Straten E, Wahli W, Kuipers F, Müller M Kersten S: The fasting-induced adipose factor/angiopoietin-like protein $\mathbf{4}$ is physically associated with lipoproteins and governs plasma lipid levels and adiposity. J Biol Chem 2006, 281(2):934-44.

25. Ashburner M, Ball CA, Blake JA, Botstein D, Butler $\mathrm{H}$, Cherry JM, Davis AP, Dolinski K, Dwight SS, Eppig JT, Harris MA, Hill DP, Issel-Tarver L, Kasarskis A, Lewis S, Matese JC, Richardson JE, Ringwald M, Rubin GM, Sherlock G: Gene ontology: tool for the unification of biology. The Gene Ontology Consortium. Nat Genet 2000, 25:25-9.

26. Falcon S, Gentleman R: Using GOstats to test gene lists for GO term association. Bioinformatics 2007, 23(2):257-8.

27. Christoffels VM, Habets PE, Franco D, Campione M, de Jong, F, Lamers WH, Bao ZZ, Palmer S, Biben C, Harvey RP, Moorman AF: Chamber formation and morphogenesis in the developing mammalian heart. Dev Biol 2000, 223(2):266-78.

28. Chen JN, Fishman MC: Zebrafish tinman homolog demarcates the heart field and initiates myocardial differentiation. Development 1996, 122(12):3809-16.

29. Schwinger RHG, Bundgaard H, Müller-Ehmsen J, Kjeldsen K: The Na, K-ATPase in the failing human heart. Cardiovasc Res 2003, 57(4):913-20.

30. Wehrens XHT, Lehnart SE, Huang F, Vest JA, Reiken SR, Mohler PJ, Sun J, Guatimosim S, Song LS, Rosemblit N, D'Armiento JM, Napolitano C, Memmi M, Priori SG, Lederer WJ, Marks AR: FKBP12.6 deficiency and defective calcium release channel (ryanodine receptor) function linked to exercise-induced sudden cardiac death. Cell 2003, 113(7):829-40.

31. Frank KF, Bölck B, Erdmann E, Schwinger RHG: Sarcoplasmic reticulum Ca2+-ATPase modulates cardiac contraction and relaxation. Cardiovasc Res 2003, 57:20-7.

32. BeckEJ, Bowlby M, An WF, Rhodes KJ, Covarrubias M: Remodelling inactivation gating of Kv4 channels by KChIP1, a small-molecular-weight calcium-binding protein. J Physio/ 2002, 538(Pt 3):691-706

33. Wickenden AD, Kaprielian R, Kassiri Z, Tsoporis JN, Tsushima R, Fishman GI, Backx PH: The role of action potential prolongation and altered intracellular calcium handling in the pathogenesis of heart failure. Cardiovasc Res 1998, 37(2):312-23.

34. Dyck JR, Barr AJ, Barr RL, Kolattukudy PE, Lopaschuk GD: Characterization of cardiac malonyl-CoA decarboxylase and its putative role in regulating fatty acid oxidation. Am J Physiol 1998, 275(6 Pt 2):H2122-9.

35. Sans CL, Satterwhite DJ, Stoltzman CA, Breen KT, Ayer DE: MondoA-MIx heterodimers are candidate sensors of cellular energy status: mitochondrial localization and direct regulation of glycolysis. $\mathrm{Mol}$ Cell Biol 2006, 26(13):4863-71.

36. Totland GK, Madsen L, Klementsen B, Vaagenes H, Kryvi H, Frøyland L, Hexeberg S, Berge RK: Proliferation of mitochondria and gene expression of carnitine palmitoyltransferase and fatty acyl-CoA oxidase in rat skeletal muscle, heart and liver by hypolipidemic fatty acids. Biol Cell 2000, 92(5):317-29.

37. Kleveland EJ, Ruyter B, Vegusdal A, Sundvold H, Berge RK, Gjøen T: Effects of 3-thia fatty acids on expression of some lipid related genes in Atlantic salmon (Salmo salar L.). Comp Biochem Physiol B, Biochem Mol Biol 2006, 145(2):239-48.

38. Hafstad AD, Khalid AM, Hagve M, Lund T, Larsen TS, Severson DL, Clarke K, Berge RK, Aasum E: Cardiac peroxisome proliferator-activated receptor-alpha activation causes increased fatty acid oxidation, reducing efficiency and post-ischaemic functional loss. Cardiovasc Res 2009, 83(3):519-26.

39. Wagner N, Jehl-Piétri C, Lopez P, Murdaca J, Giordano C, Schwartz C, Gounon P, Hatem SN, Grimaldi P, Wagner KD: Peroxisome proliferator-activated receptor beta stimulation induces rapid cardiac growth and angiogenesis via direct activation of calcineurin. Cardiovasc Res 2009, 83:61-71.

40. Narkar VA, Downes M, Yu RT, Embler E, Wang YX, Banayo E, Mihaylova MM, Nelson MC, Zou Y, Juguilon H, Kang H, Shaw RJ, Evans RM: AMPK and PPARdelta agonists are exercise mimetics. Cell 2008, 134(3):405-15. 
41. Xu J, Gong NL, Bodi I, Aronow BJ, Backx PH, Molkentin JD: Myocyte enhancer factors $2 \mathrm{~A}$ and $2 \mathrm{C}$ induce dilated cardiomyopathy in transgenic mice. J Biol Chem 2006, 281(14):9152-62.

42. Sharifpanah F, Wartenberg M, Hannig M, Piper HM, Sauer H: Peroxisome proliferator-activated receptor alpha agonists enhance cardiomyogenesis of mouse ES cells by utilization of a reactive oxygen species-dependent mechanism. Stem Cells 2008, 26:64-71.

43. Poppe $T$, Johansen R, Gunnes $G$, Tørud B: Heart morphology in wild and farmed Atlantic salmon Salmo salar and rainbow trout Oncorhynchus mykiss. Dis Aquat Org 2003, 57(1-2):103-8.

44. Tørud B, Hillestad M: “Hjerte-rapporten” Rapport om hjertelidelser hos laks og regnbueørret. 2004:1-69.

[www.fiskerifond.no/files/projects/attach/hjerterapporten.pdf].

45. Asiedu DK, Frøyland L, Vaagenes H, Lie O, Demoz A, Berge RK: Long-term effect of tetradecylthioacetic acid: a study on plasma lipid profile and fatty acid composition and oxidation in different rat organs. Biochim Biophys Acta 1996, 1300(2):86-96.

46. Gjøen T, Kleveland EJ, Moya-Falcón C, Frøystad MK: Effects of dietary thia fatty acids on lipid composition, morphology and macrophage function of Atlantic salmon (Salmo salar L.) kidney. Comp Biochem Physiol B, Biochem Mol Biol 2007, 148:103-11.

47. Alne H, Oehme M, Thomassen M, Terjesen B, Rørvik KA: Reduced growth, condition factor and body energy levels in Atlantic salmon Salmo salar L. during their first spring in the sea. Aquacult Res 2010, 42(2):248-259.

48. Bligh EG, Dyer WJ: A rapid method of total lipid extraction and purification. Can J Biochem Physiol 1959, 37:911-917.

49. Dates M: General analytical procedures. In Techniques in Lipidology. Edited by Kates M. Elsevier; 1986

50. R Development CoreTeam: R: A Lanquage and Environment for Statistical Computing. Vienna: R Foundation for Statistical Computing; 2009. http:// www.R-project.org. [ISBN 3-900051-07-0].

51. Gentleman RC, Carey VJ, Bates DM, et al.: Bioconductor: Open software development for computational biology and bioinformatics. Genome Biology 2004, 5:R80.

52. Bolstad BM, Irizarry RA, Astrand M, Speed TP: A comparison of normalization methods for high density oligonucleotide array data based on variance and bias. Bioinformatics 2003, 19(2):185-93.

53. Smyth GK: Linear models and empirical bayes methods for assessing differential expression in microarray experiments. Stat Appl Genet Mol Biol 2004, 3:Article3.

54. Benjamini $\mathrm{Y}$, Yosef $\mathrm{H}$ : Controlling the false discovery rate: a practical and powerful approach to multiple testing. J Roy Stat Soc B 1995, 57:289-300

55. Conesa A, Götz S, García-Gómez JM: Blast2GO: a universal tool for annotation, visualization and analysis in functional genomics research. Bioinformatics 2005, 21(18):3674-6

56. Culhane AC, Thioulouse J, Perrière G, Higgins: MADE4: an R package for multivariate analysis of gene expression data. Bioinformatics 2005 , 21(11):2789-90

57. Olsvik PA, Lie KK, Jordal AEO, Nilsen TO, Hordvik I: Evaluation of potential reference genes in real-time RT-PCR studies of Atlantic salmon. BMC Mol Biol 2005, 6:21.

58. Wickham H: Ggplot: Elegant graphics for data analysis. New York: Springer; 2009

59. Sarkar D: Lattice: Multivariate Data Visualization with R. New York: Springer; 2008

60. Moriya Y, Itoh M, Okuda S, Yoshizawa AC, Kanehisa M: KAAS: an automatic genome annotation and pathway reconstruction server. Nucleic Acids Res 2007, 35(Web Server issue):W182-5.

doi:10.1186/1471-2164-13-180

Cite this article as: Grammes et al: Genome wide response to dietary tetradecylthioacetic acid supplementation in the heart of Atlantic Salmon (Salmo salar L). BMC Genomics 2012 13:180.

\section{Submit your next manuscript to BioMed Central and take full advantage of:}

- Convenient online submission

- Thorough peer review

- No space constraints or color figure charges

- Immediate publication on acceptance

- Inclusion in PubMed, CAS, Scopus and Google Scholar

- Research which is freely available for redistribution 